\title{
Animal Models of Ischemic Stroke. Part Two: Modeling Cerebral Ischemia
}

\author{
Marco Bacigaluppi ${ }^{1-3}$, Giancarlo Comi ${ }^{3}$ and Dirk M. Hermann*,1,4 \\ ${ }^{I}$ Department of Neurology, University Hospital Zurich (USZ), Zurich, Switzerland; ${ }^{2}$ Neuroimmunology Unit, DIBIT and \\ ${ }^{3}$ Institute of Experimental Neurology (INSPE), IRCCS San Raffaele Hospital, Milan, Italy; ${ }^{4}$ Department of Vascular \\ Neurology, Dementia and Ageing Research, Neurology Clinic, University Hospital Essen, Essen, Germany.
}

\begin{abstract}
Animal models of stroke provide an essential tool for the understanding of the complex cellular and molecular pathophysiology of stroke and for testing novel recanalyzing, neuroprotective, neuroregenerative or anti- inflammatory drugs in pre- clinical setting. Since the first description of the distal occlusion of the middle cerebral artery (MCA) in rats, different techniques and methods to induce focal and global ischemia of the brains have been developed and optimized. The different models, ranging from proximal to distal MCA occlusion to embolic and photothrombotic stroke vary widely in their ability to model human disease and in their application to the study of cell death, inflammation and neural repair. In the first part of the review animal models developed for studying stroke related risk factors are described while this section discusses specific models that have been created for mimicking different types of stroke, focal and global ischemia in an experimental setup. Advantages and limits and the potential of the diverse models for the study of novel therapies as well as for the study of basic pathophysiological mechanisms are explored.
\end{abstract}

Keywords: Animal Model, focal and global ischemia, middle cerebral artery occlusion (MCAo), pathophysiology, stroke.

\section{INTRODUCTION}

From the late 1970s, animal models of cerebral ischemia were developed with the aim of identifying mechanisms that cause tissue damage and to provide the basis for the development, at a preclinical level, of new therapies for stroke. Several recent animal models have been designed specifically to address specific risk factors, to determine neural repair processes, to test new neuroprotective and recanalizing strategies. Today reliable animal models for stroke are available in a variety of species including primates, pigs, sheep, dogs, cats, mongolian gerbils, rabbits, rats and mice.

Although the use and development of primate and higher mammal stroke models is a very important goal, small animals are actually privileged at a preclinical level for the following reasons: they are well-suited for ischemic strokes, physiological variables can reasonably be monitored and sufficient numbers for statistical analysis can be reached without excessive costs [1]. In stroke studies the rat is the most commonly used animal because of its size that allows easy monitoring of the physiologic variables and handling of vascular structures.

Nonetheless since the mouse is the best-characterized animal in genetics and molecular biology, many transgenic animals being available in this species, an increasing number of stroke studies were carried out in mice from the 1990s onwards $[2,3]$.

*Address correspondence to this author at the Department of Vascular Neurology, Dementia and Ageing Research, Neurology Clinic, University Hospital Essen, Hufelandstr. 55, D-45122 Essen, Germany; Tel: +49-201-723 2180; Fax: +49-201-723-5534; E-mail: dirk.hermann@uk-essen.de
Stroke models can be divided into two main categories:

1. Models to study how risk factors (both environmental and genetic) may contribute to vascular damage that ultimately leads to stroke (i.e. models of atherosclerosis, hypercholesterolemia, hyper-homocysteinemia, arterial hypertension and single-gene disorders associated with stroke such as CADASIL) and therapeutical approaches to prevent stroke events- (These models are discussed in the first part of the review: "Part one: Modeling risk factors").

2. Models for the study of the pathophysiological consequences of stroke, and for testing therapeutical strategies (recanalyzing, neuroprotective and neuroreparative approaches). These latter are further subdivided in models of focal and global cerebral ischemia.

Animal models of tissue injury in stroke are designed to generate reproducible infarcts in a high throughput manner with a minimum of surgical manipulation to determine mechanisms of cell death and to test novel drugs as recanalyzing, neuroprotective, neuroregenerative and anti- inflammatory therapies [1].

The various methods to induce strokes in rodents reflect the complexity of the disease, and the need of different paradigms to optimize the study of therapeutical approaches.

\section{MODELS FOR THE STUDY OF THE PATHOPHYSI- OLOGY AND THERAPIES FOR STROKE}

Models of cerebral ischemia can be separated into focal and global ischemia models. Focal ischemia is characterized by a reduction of cerebral blood flow in a distinct region of the brain, whereas in global ischemia the reduction of blood flow affects the entire brain or forebrain $[4,5]$. In focal cere- 
bral ischemia, either an artery or vein is occluded mechanically or by cerebral thromboembolism.

\section{Models for Inducing Focal Cerebral Ischemia}

Stroke caused by an acute cerebral vessel occlusion can be reproduced by different techniques, namely by mechanical occlusion of either the proximal middle cerebral artery (pMCAo) (large vessel occlusion) or distal MCA (dMCAo) (small vessel occlusion), or by thrombotic occlusion either via injection of blood clots or thrombin into the MCA or by photo-thrombosis after intravenous injection of Rose Bengal. Besides models for arterial stroke also animal models for cerebral venous sinus thrombosis, a rare form of stroke that results from thrombosis of the dural venous sinuses, have been developed.

\section{a. Mechanical occlusion of the MCA}

pMCAo models belong to the most frequently used procedures in stroke research. pMCAo is usually induced by direct mechanical occlusion, most often through the insertion of a silicon-coated nylon suture into the internal carotid artery that is subsequently advanced to the circle of Willis to occlude the MCA at its origin. The severity of ischemic injury can be modeled by leaving the suture filament in place either transiently for a variable duration of time (time usually ranges between $30-120 \mathrm{~min}$ ) before the suture is removed to allow tissue reperfusion. In case of permanent pMCAo the suture is left in place and no reperfusion is allowed. Shortlasting pMCAo causes selective neuronal death in the lesionsided striatum, expression of heat shock proteins, immediate early gene expression and induction of apoptotic signal pathways in the overlying cortex [6,7]. Longer durations of occlusion instead result in brain infarcts that involve both the striatum and cortex [8], and may be associated with some animal mortality in case of edema formation. Intracranial hypertension due to edema is frequently observed in animals, in which brain injury develops in deep brain structures comprising the thalamus and midbrain. One disadvantage with this model, i.e. the intraluminal MCA occlusion, is that injuring these structures is unavoidable in some. However altogether pMCAo has the advantage of its high reproducibility and that it does not require craniotomy (avoiding possible, although rare complications) and that the resulting focal brain damages are similar to those occurring in human stroke $[9,10]$.

Distal vascular occlusions, dMCAo, can be on the other hand performed through a craniotomy to directly expose and manipulate superficial MCA branches. Compared to pMCAo, the damage with distal occlusions is more restricted as the occluded vessel is superficial. Usually the MCA is occluded either transiently or permanently distal to the origin of the lenticulostriate branches by means of a clip or by electrocoagulation [11, 12]. Deep structures of the brain as thalamus hypothalamus, hippocampus and midbrain are spared but the procedure, though seldom, may be associated with some complications like side effects of the craniotomy as subarachnoidal bleeding, cerebral infection, mechanical induction of spreading depression and cerebrospinal fluid leakage [1].

dMCAo can also be combined with either ipsilateral permanent or bilateral transient common carotid artery occlusion. Ischemic damage may involve the frontal, parietal and temporal cortex. With these latter technique, the underlying white matter together with a small area of the lateral striatum may be involved as well [13-15].

As selective damage in the cortex can be reproduced without the involvement of deep structures the model of dMCAo is particularly suited for studying neuroplasticity rearrangements after stroke and neuroregeneration cues.

\section{b. Thromboembolic Models}

As human stroke is most frequently caused by cerebral thromboembolism, a number of animal models has been developed that closely mimic the embolic occlusion of brain vessels. Embolic strokes can be induced in animals through injection of large-sized synthetic macrospheres (300-400 $\mu \mathrm{m}$ diameter) or small-sized microspheres (less than $50 \mu \mathrm{m}$ ) into the internal carotid artery. In the first case, large infarcts similar to those produced by the permanent occlusion of the MCA are induced. In the latter case, smaller, multifocal infarcts can occur $[16,17]$.

To study thrombolytic therapies (a clinically very relevant therapeutical issue), a third model of vascular occlusion has been developed using autologous blood clots that are injected directly into the internal carotid artery [18-22]. Embolic stroke models produce relatively variable infarcts that make it more difficult to test neuroprotective therapies; nevertheless they are well suited to study reperfusion therapies [23].

Very recently a novel mouse model of in situ thromboembolic stroke has been described [24]. Generation of an autologous thrombus directly into the MCA induced by the local injection of purified thrombin resulted in very reproducible ischemic injury volumes. In this thromboembolic model recombinant tissue plasminogen activator (rtPA) delivery resulted in a significant reduction of brain injury. Such local delivery techniques might be applied in the previously described models to overcome the low reproducibility of ischemic infarcts [25]. Nonetheless the positioning of intraarterial catheters for thrombus injection might produce endovascular injuries and subsequent inflammatory processes. Whether such bystander injuries can be prevented by refinements of clot delivery techniques need to be assessed.

Unfortunately, thromboembolic stroke models have achieved limited significance in neuroprotection research in the past, due to their variability, thus accounting for the translation failure of animal data to humans. In fact, mechanical occlusion models poorly reflected the hemodynamic aspects of thrombolytic reperfusion, which potentially might lead to an altered responsiveness of the brain tissue to neuroprotective treatments. The latter idea is supported by observations regarding the progression of metabolic disturbances and energy failure after cerebral thromboembolism followed by thrombolysis in mice [26], which differed from intraluminal MCA occlusion findings $[7,8,27]$. It remains to be awaited whether the refinement of models might in the near future give the opportunity to study more precisely the effect of recanalizing strategies in animal models.

\section{c. The endothelin and the photothrombosis model}

As the more commonly used models (suture model and distal middle cerebral artery occlusion) are often technically challenging, simpler models to induce a focal ischemic le- 
sion have been developed as an alternative. One of these methods consists in the intracerebral injection of vasoconstrictor substances as endothelin-1 (ET-1) that causes a lesion by reducing acutely the blood flow in the circumscribed injected area [28]. Although this has been reported as a simple and reproducible method of focal ischemia in rats [28, 29] the intracerebral injection of ET-1 in mice often does not produce a lesion [30]. In mice it is therefore necessary to combine ET-1 injection with either common carotid artery (CCA) occlusion or $N(G)$-nitro-larginine methyl ester (1NAME) injection or both to produce a reproducible lesion resulting also in a significant motor deficit. This model has therefore the advantage of being technically easier and faster than other rat stroke models however it does not confer other advantages (no precise localization of the lesion to the site of injection, similar mortality rate as other stroke models) [30].

Another technique to induce localized stroke consists of the photothrombosis of an injected photosensitive dye. This is induced by the trans-cranial illumination of the brain after the systemic delivery of a photosensitive dye (Rose Bengal), obtaining the coagulation of the irradiated tissue [31]. This model has the advantage that the region of ischemia can be predefined and highly circumscribed, opening the possibility to coagulate distinct cortical areas with stereotactical precision. The disadvantages of this model are the nonphysiologic insult (photocoagulation) that creates a lesion with a small ischemic penumbra compared to other focal stroke models as described above. Further, because of the damage of the vessels induced by the "photothrombosis",substantial local vasogenic edema forms early after infarction [1].

\section{d. Cerebral Venous Thrombosis Models}

Cerebral veins and sinus thrombosis (CVT) is a distinct cerebrovascular disorder that affects young adults and children more often than arterial stroke. The acute or chronic thrombosis of cerebral veins and/or sinuses is the underlying cause. During the past decade, increased awareness of the diagnosis, improved neuroimaging techniques, and more effective treatments have remarkably improved the prognosis [32]. The first report on animal models of CVT is of the year 1836 in a rabbit by tying both jugular veins [33]. Since then various other animal models of CVT have been developed in cats, pigs, dogs, rabbits and rodents [34]. However rat models are more reliable because of their anatomy of the cerebral brains and sinuses. CVT can be induced by different methods: occlusion of the superior sagittal sinus can be induced by direct injection of various chemical substances (cyanoacrylate, ethanolamine or other sclerosing agents) into the sinus. Other techniques make use of direct mechanical or heat damage to the superior sagittal sinus. Another rat model of CVT is induced by the photochemical coagulation of the dorsal cerebral veins after injection of Rose Bengal [35]. Unfortunately, the induction of CVT is often insufficient to mimic neuropathological consequences of CVT [2].

\section{Models for Inducing Global Cerebral Ischemia}

Global cerebral ischemia, characterized by the critical reduction of cerebral blood flow in the whole brain, induces selectively neuronal injury in the CA1 region of the hippocampus as long as the duration of ischemia is limited. Upon extension of ischemia, other brain areas get involved, and at a critical duration a so-called no-reflow phenomenon takes place in which the restoration of blood flow is unsuccessful [36-38].

Models of global cerebral ischemia are usually used to study brain damage that occurs in cardio-circulatory resuscitation.

Global ischemia can be induced by means of different approaches. The so called 'four vessel occlusion method' (4VO) consists of a reversible CCA occlusion, which, combined with permanent interruption of the vertebral arteries via electro cauterization, results in bilateral forebrain and brainstem ischemia with a highly predictable brain damage [39].

As alternative to the $4 \mathrm{VO}$ method, global ischemia can also be induced by the occlusion of the two common carotid arteries, i.e., by two vessel occlusion (2VO) together with induction of hypotension for a limited time period. In this forebrain ischemia model, selective injury in the CA1 of the hippocampus, the caudate putamen and neocortex is observed [40].

The mongolian Gerbils, small mammal of the order Rodentia, are widely used in models of chronic or transient forebrain ischemia due to specie-specific incomplete circle of Willis, i.e. absence of communicating arteries between the middle and posterior cerebral arteries. For this anatomic peculiarity chronic bilateral common carotid stenosis using steel coils produces prolonged hypoperfusion, with a $25 \%$ reduction in cerebral blood flow [41]. On neuropathology the chronic hypoperfusion reflects as small necrotic foci in the cortex and the basal ganglia, accompanied by intense gliosis and small vessel proliferation. If the common carotid arteries are transiently occluded (10 minutes long) neuronal death in the hippocampus is observed [42]. These changes are also accompanied by various behavioral impairments as a spatial learning and memory deficits that varies with the degree and the duration of hypoperfusion. However the gerbils might present various problems when used in models for global cerebral ischemia, as many animals have not a totally incomplete circle of Willis, i.e. small communicating arteries give collateral supply to the forebrain [43].

Another technique for inducing a global cerebral ischemia that is very close to that occurring in the human during cardiac arrest consists in the induction of ventricular fibrillation followed shortly thereafter by defibrillation combined with chest compression and administration of epinephrine. This technique is commonly performed in large animals species due to its complexity and the fact that complete intensive care treatment must be ensured during the first couple of days after resuscitation $[44,45]$ even though it has also been successfully adapted to small rodents [46].

Models of global cerebral ischemia have widely been used in large mammals as the dog and the pig, since cardiocirculatory variables can be controlled similar to humans in these animals. Mouse models were initially less common as they were hampered by a high mortality rate and frequent complication that included most often seizures [4]. Due the need to use transgenic or knockout mutant mice, global ischemia was recently induced in mice by the bilateral occlusion of the common carotid artery in association with controlled ventilation [47] and, clinically even more relevant, by 
Table 1. Animal Models of Stroke for Studying Pathophysiology and Therapy

\begin{tabular}{|l|l|l|}
\hline Model for & Model name & Animal species available \\
\hline \hline \multirow{3}{*}{ Focal cerebral ischemia } & Proximal middle cerebral artery occlusion & Dog, cat rabbit, rodents \\
\cline { 2 - 3 } & Distal middle cerebral artery occlusion & Nonhuman primate, dog, cat, pig, rabbit, rodents \\
\cline { 2 - 3 } & Thromboembolic MCA occlusion & Rodents \\
\cline { 2 - 3 } & Endothelin model & Rodents \\
\cline { 2 - 3 } & Photothrombosis model & Rodents \\
\hline \multirow{2}{*}{ Gerebral venous thrombosis } & Cerebral venous thrombosis & Dog, cats, pigs, rabbits, rodents \\
\cline { 2 - 3 } & Four vessel occlusion & Dog, pig, rodents \\
\cline { 2 - 3 } & Two vessel occlusion & Dog, pig, rodents \\
\cline { 2 - 3 } & Cardiac arrest and resuscitation & Dog, pig, rodents \\
\hline
\end{tabular}

the induction of cardiac arrest (8-10 min), followed by cardio-pulmonary resuscitation. This latter model is in fact characterized by a selective injury of neurons in the hippocampus and striatum [48].

Animal models discussed have been resumed in Table $\mathbf{1 .}$

\section{CONCLUSION: RESEARCH RECOMMENDATIONS AND ETHICAL ISSUES}

Research projects should be designed on the basis of state-of-the-art recommendations on preclinical and clinical studies, e.g., made by the Stroke Therapy Academic Industry Roundtable (STAIR), [49-51] that aim to provide common standards and to increase the success of drug developments. These recommendations request experimental works to be performed over species and sex barriers, providing doseresponse relationships and time windows for drug application before studies should enter clinical testing. The inclusion of physiological measurements and functional readouts in addition to morphological data analysis for animal studies is also recommended. One major problem that is nonetheless present and difficult to overcome is that stroke research is mostly conducted in young laboratory animals and thus does not consider that the vast majority of strokes in humans that occur in elderly subjects affected by a multiplicity of other co-morbidities and risk factors, such as arterial hypertension, diabetes and atherosclerosis. The authors recognize an increasing need to include more and more experiments evaluating ischemic injury evolution in animal lines at risk for vascular injury, such as in atherosclerotic, diabetic mice or aged rats.

It must critically be mentioned though that until the present stage, not many experimental stroke studies made use of the pool of transgenic animals at vascular risk described in the first part of this review. Clearly, research is needed in that area, analyzing how the responsiveness of the brain is altered in animals in which the vascular system is compromised. Although no single animal precisely reproduces human ischemic stroke (the underlying risk factors and conditions are heterogeneous and complex), the lack of studies in that field from our view might represent an important reason for study failures in clinics. With such strategies, the authors would not be surprised if neuroprotection, which has been a problem for a considerable duration of time, would become feasible also in human patients.

\section{REFERENCES}

[1] Carmichael ST. Rodent models of focal stroke: size, mechanism, and purpose. NeuroRx 2005; 2(3): 396-409.

[2] Durukan A, Tatlisumak T. Acute ischemic stroke: overview of major experimental rodent models, pathophysiology, and therapy of focal cerebral ischemia. Pharmacol Biochem Behav 2007; 87(1): 179-97.

[3] Harper AJ. Production of transgenic and mutant mouse models. Methods Mol Med 2005; 104: 185-202.

[4] Traystman RJ. Animal models of focal and global cerebral ischemia. ILAR journal / National Research Council, Institute of Laboratory Animal Resources 2003; 44(2): 85-95.

[5] Bacigaluppi M, Hermann DM. New targets of neuroprotection in ischemic stroke. Sci World J 2008; 8: 698-712.

[6] Namura S, Zhu J, Fink K, et al. Activation and cleavage of caspase-3 in apoptosis induced by experimental cerebral ischemia. J Neurosci 1998; 18(10): 3659-68.

[7] Hermann DM, Kilic E, Hata R, Hossmann KA, Mies G. Relationship between metabolic dysfunctions, gene responses and delayed cell death after mild focal cerebral ischemia in mice. Neuroscience 2001; 104(4): 947-55.

[8] Hata R, Maeda K, Hermann D, Mies G, Hossmann KA. Evolution of brain infarction after transient focal cerebral ischemia in mice. J Cereb Blood Flow Metab 2000 Jun; 20(6): 937-46.

[9] Menzies SA, Hoff JT, Betz AL. Middle cerebral artery occlusion in rats: a neurological and pathological evaluation of a reproducible model. Neurosurgery 1992; 31(1): 100-6; discussion 6-7.

[10] Mies G, Ishimaru S, Xie Y, Seo K, Hossmann KA. Ischemic thresholds of cerebral protein synthesis and energy state following middle cerebral artery occlusion in rat. J Cereb Blood Flow Metab 1991; 11(5): 753-61.

[11] Iadecola C, Zhang F, Casey R, Nagayama M, Ross ME. Delayed reduction of ischemic brain injury and neurological deficits in mice lacking the inducible nitric oxide synthase gene. J Neurosci 1997; 17(23): 9157-64.

[12] Tamura A, Graham DI, McCulloch J, Teasdale GM. Focal cerebral ischaemia in the rat: 1: description of technique and early neuropathological consequences following middle cerebral artery occlusion. J Cereb Blood Flow Metab 1981; 1(1): 53-60.

[13] Brint S, Jacewicz M, Kiessling M, Tanabe J, Pulsinelli W. Focal brain ischemia in the rat: methods for reproducible neocortical infarction using tandem occlusion of the distal middle cerebral and ipsilateral common carotid arteries. J Cereb Blood Flow Metab 1988; 8(4): 474-85.

[14] Rubino GJ, Young W. Ischemic cortical lesions after permanent occlusion of individual middle cerebral artery branches in rats. Stroke 1988; 19(7): 870-7.

[15] Chen ST, Hsu CY, Hogan EL, Maricq H, Balentine JD. A model of focal ischemic stroke in the rat: reproducible extensive cortical infarction. Stroke 1986; 17(4): 738-43.

[16] Gerriets T, Li F, Silva MD, et al. The macrosphere model: evaluation of a new stroke model for permanent middle cerebral artery occlusion in rats. J Neurosci Methods 2003; 122(2): 201-11. 
[17] Miyake K, Takeo S, Kaijihara H. Sustained decrease in brain regional blood flow after microsphere embolism in rats. Stroke 1993; 24(3): 415-20.

[18] Kilic E, Hermann DM, Hossmann KA. A reproducible model of thromboembolic stroke in mice. Neuroreport 1998 ; 9(13): 296770.

[19] Kilic E, Hermann DM, Hossmann KA. Recombinant tissue plasminogen activator reduces infarct size after reversible thread occlusion of middle cerebral artery in mice. Neuroreport $1999 ; 10(1)$ : 107-11.

[20] Brinker G, Franke C, Hoehn M, Uhlenkuken U, Hossmann KA. Thrombolysis of cerebral clot embolism in rat: effect of treatment delay. Neuroreport 1999; 10(16): 3269-72.

[21] Brinker G, Pillekamp F, Hossmann KA. Brain hemorrhages after rt-PA treatment of embolic stroke in spontaneously hypertensive rats. Neuroreport 1999; 10(9): 1943-6.

[22] Busch E, Kruger K, Allegrini PR, et al. Reperfusion after thrombolytic therapy of embolic stroke in the rat: magnetic resonance and biochemical imaging. J Cereb Blood Flow Metab 1998; 18(4): 407-18.

[23] Kilic E, Hermann DM, Hossmann KA. Recombinant tissueplasminogen activator-induced thrombolysis after cerebral thromboembolism in mice. Acta Neuropathologica 2000; 99(3): 219-22.

[24] Orset C, Macrez R, Young AR, et al. Mouse model of in situ thromboembolic stroke and reperfusion. Stroke 2007; 38(10): 2771-8.

[25] Kudo M, Aoyama A, Ichimori S, Fukunaga N. An animal model of cerebral infarction. Homologous blood clot emboli in rats. Stroke 1982; 13(4): 505-8.

[26] Hara T, Mies G, Hossmann KA. Effect of thrombolysis on the dynamics of infarct evolution after clot embolism of middle cerebral artery in mice. J Cereb Blood Flow Metab 2000; 20(10): 148391

[27] Hata R, Maeda K, Hermann D, Mies G, Hossmann KA. Dynamics of regional brain metabolism and gene expression after middle cerebral artery occlusion in mice. J Cereb Blood Flow Metab 2000; 20(2): 306-15

[28] Fuxe K, Cintra A, Andbjer B, Anggard E, Goldstein M, Agnati LF. Centrally administered endothelin-1 produces lesions in the brain of the male rat. Acta Physiol Scand 1989; 137(1): 155-6.

[29] Kurosawa M, Fuxe K, Hallstrom A, Goiny M, Cintra A, Ungerstedt $\mathrm{U}$. Responses of blood flow, extracellular lactate, and dopamine in the striatum to intrastriatal injection of endothelin-1 in anesthetized rats. J Cardiovaspharmacology. 1991; 17(Suppl 7): S340-2.

[30] Horie N, Maag AL, Hamilton SA, Shichinohe H, Bliss TM, Steinberg GK. Mouse model of focal cerebral ischemia using endothelin-1. J Neurosci Methods 2008; 173(2): 286-90.

[31] Watson BD, Dietrich WD, Busto R, Wachtel MS, Ginsberg MD. Induction of reproducible brain infarction by photochemically initiated thrombosis. Ann Neurol 1985; 17(5): 497-504

[32] Stam J. Thrombosis of the cerebral veins and sinuses. N Engl J Med 2005 28; 352(17): 1791-8.

[33] Brock RC. Astley Cooper and carotid artery ligation. Guy's Hosp Rep 1968; 117(3): 219-24.

[34] Schaller B, Graf R, Wienhard K, Heiss WD. A new animal model of cerebral venous infarction: ligation of the posterior part of the superior sagittal sinus in the cat. Swiss Med Wkly 2003; 133(2930): 412-8.
[35] Nakase H, Kakizaki T, Miyamoto K, Hiramatsu K, Sakaki T. Use of local cerebral blood flow monitoring to predict brain damage after disturbance to the venous circulation: cortical vein occlusion model by photochemical dye. Neurosurgery 1995 37(2): 280-5; discussion 5-6.

[36] Bottiger BW, Krumnik1 JJ, Gass P, Schmitz B, Motsch J, Martin E. The cerebral 'no-reflow' phenomenon after cardiac arrest in ratsinfluence of low-flow reperfusion. Resuscitation 1997; 34(1): 7987.

[37] Fischer M, Hossmann KA. No-reflow after cardiac arrest. Intens Care Med 1995; 21(2): 132-41.

[38] Ames A, 3rd, Wright RL, Kowada M, Thurston JM, Majno G. Cerebral ischemia. II. The no-reflow phenomenon. Am J Pathol 1968; 52(2): 437-53

[39] Pulsinelli WA, Brierley JB. A new model of bilateral hemispheric ischemia in the unanesthetized rat. Stroke 1979; 10(3): 267-72.

[40] Smith ML, Bendek G, Dahlgren N, Rosen I, Wieloch T, Siesjo BK. Models for studying long-term recovery following forebrain ischemia in the rat. 2. A 2-vessel occlusion model. Acta Physiol Scand 1984; 69(6): 385-401.

[41] Kudo T, Takeda M, Tanimukai S, Nishimura T. Neuropathologic changes in the gerbil brain after chronic hypoperfusion. Stroke 1993; 24(2): 259-64; discussion 65.

[42] Giuliani D, Leone S, Mioni C, et al. Broad therapeutic treatment window of [Nle(4), D-Phe(7)]alpha-melanocyte-stimulating hormone for long-lasting protection against ischemic stroke, in Mongolian gerbils. Eur J Pharmacol 2006; 538(1-3): 48-56.

[43] Seal JB, Buchh BN, Marks JD. New variability in cerebrovascular anatomy determines severity of hippocampal injury following forebrain ischemia in the Mongolian gerbil. Brain Res 2006; 10731074: 451-9.

[44] Berkowitz ID, Gervais H, Schleien CL, Koehler RC, Dean JM, Traystman RJ. Epinephrine dosage effects on cerebral and myocardial blood flow in an infant swine model of cardiopulmonary resuscitation. Anesthesiology 1991; 75(6): 1041-50.

[45] Eleff SM, Maruki Y, Monsein LH, Traystman RJ, Bryan RN, Koehler RC. Sodium, ATP, and intracellular $\mathrm{pH}$ transients during reversible complete ischemia of dog cerebrum. Stroke 1991; 22(2): 233-41

[46] Kofler J, Hattori K, Sawada M, et al. Histopathological and behavioral characterization of a novel model of cardiac arrest and cardiopulmonary resuscitation in mice. J Neurosci Methods 2004; 136(1): 33-44.

[47] Murakami K, Kondo T, Kawase M, Chan PH. The development of a new mouse model of global ischemia: focus on the relationships between ischemia duration, anesthesia, cerebral vasculature, and neuronal injury following global ischemia in mice. Brain Res1998; 780(2): 304-10.

[48] Krieglstein J, Klumpp S. Pharmacology of cerebral ischemia 2000 Stuttgart: Medpharm Scientific 2000.

[49] Recommendations for standards regarding preclinical neuroprotective and restorative drug development. Stroke 1999; 30(12): 27528.

[50] Fisher M. Recommendations for advancing development of acute stroke therapies: stroke therapy academic industry roundtable 3 . Stroke 2003; 34(6): 1539-46.

[51] Recommendations for clinical trial evaluation of acute stroke therapies. Stroke 2001; 32(7): 1598-606 